\title{
COSTS OF PLASTICITY IN FORAGING CHARACTERISTICS OF THE CLONAL PLANT RANUNCULUS REPTANS
}

\author{
Mark van Kleunen, ${ }^{1}$ Markus Fischer, and Bernhard Schmid \\ Institut für Umweltwissenschaften, Universität Zürich, Winterthurerstrasse 190, CH-8057 Zürich, Switzerland \\ ${ }^{1}$ E-mail: vkleunen@uwinst.unizh.ch
}

\begin{abstract}
In clonal plants, evolution of plastic foraging by increased lengths of leaves and internodes under unfavourable conditions may be constrained by costs and limits of plasticity. We studied costs and limits of plasticity in foraging characteristics in 102 genotypes of the stoloniferous herb Ranunculus reptans. We grew three replicates of each genotype with and three without competition by the naturally co-occuring grass Agrostis stolonifera. We used regression and correlation analyses to investigate potential costs of plasticity in lengths of leaves and stolon internodes, developmental instability costs of these traits, and a developmental range limit of these traits. We used randomization procedures to control for spurious correlations between parameters calculated from the same data. Under competition the number of rosettes, rooted rosettes, and flowers was $58 \%, 40 \%$, and $61 \%$ lower, respectively, than in the absence of competition. Under competition lengths of leaves and stolon internodes were $14 \%$ and $6 \%$ smaller, respectively, than in the absence of competition. We detected significant costs of plasticity in stolon internode length in the presence of competition when fitness was measured in terms of the number of rosettes and the number of flowers (selection gradients against plasticity were 0.250 and 0.214 , respectively). Within-environment variation (SD) in both foraging traits was not positively correlated with the corresponding plasticity, which indicates that there were no developmental instability costs. More plastic genotypes did not have less extreme trait values than less plastic genotypes for both foraging traits, which indicates that there was no developmental range limit. We conclude that in $R$. reptans costs of plasticity more strongly constrain evolution of foraging in the horizontal plane (i.e., stolon internode length) than in the vertical plane (i.e., leaf length).
\end{abstract}

Key words. - Clonal growth, costs of plasticity, developmental stability, foraging, genetic variation, Ranunculus reptans, spurious correlations.

Plants, especially spreading clonal plants, are likely to experience spatial environmental heterogeneity. As long as clone parts remain connected, this heterogeneity among clone parts may be alleviated by the exchange of resources (Pitelka and Ashmun 1985). Moreover, many clonal plants have the potential for selective ramet placement in benign microhabitats by means of plasticity in branching intensity and internode lengths of stolons or rhizomes (Harper 1981; Hutchings and de Kroon 1994). Although such "foraging' responses in the horizontal direction may be beneficial, the magnitude of plasticity in internode length of some clonal plants seems to be low in comparison to plasticity in leaf length (de Kroon and Hutchings 1995; Huber 1996; Huber et al. 1998), suggesting that the evolution of plasticity in internode length may be constrained. The evolution of plasticity may be constrained by costs and limits of plasticity; as a consequence, specialist genotypes or intermediately plastic ones may evolve instead of highly plastic generalist genotypes (van Tienderen 1991, 1997; Moran 1992; León 1993; Padilla and Adolph 1996; Schlichting and Pigliucci 1998).

Here costs of plasticity are defined as the reduction in fitness of more plastic genotypes in comparison to less plastic genotypes when they produce the same phenotype in a focal environment (DeWitt 1998; DeWitt et al. 1998). Such a genetic trade-off between plasticity and fitness may arise for several reasons. More plastic genotypes may have higher energetic costs of maintaining the developmental and physiological machinery necessary for plasticity, producing inducible structures, or information acquisition (DeWitt 1998; DeWitt et al. 1998). Furthermore, more plastic genotypes may be developmentally less stable than less plastic genotypes (i.e., have developmental instability costs); as a consequence, their fitness may be reduced (Møller 1997; DeWitt 1998; DeWitt et al. 1998).

Because more plastic genotypes may have extra developmental "baggage"' to carry, they may not have as extreme trait values as less plastic genotypes (Wilson and Yoshimura 1994). Therefore, the evolution of plasticity may be limited by a narrower developmental range of more plastic genotypes compared with less plastic genotypes (DeWitt 1998; DeWitt et al. 1998).

Detection of costs and limits is most likely for adaptive plasticity because selection will strongly act against costly nonadaptive plasticity (DeWitt 1998). Furthermore, plasticity should be studied with regard to ecologically relevant factors, because only under these conditions may plasticity be adaptive. Possibly the best-known examples of adaptive plasticity in plants are their elongation of leaves and internodes in response to vegetation shade (Smith 1982; Schmitt and Wulff 1993; Schmitt et al. 1995; van Hinsberg 1996; Schlichting and Pigliucci 1998). However, under natural conditions vegetation shade is imposed by competing plants, and therefore leaf and internode elongation may also be constrained by resource limitation (van Kleunen et al. 2000). Nevertheless, long leaves and internodes are likely to be adaptive under competition.

Several of these potential costs and limits of plasticity can be estimated by regression and correlation analyses if data on a large number of families or genotypes grown in two or more different environments are available (van Tienderen 1991, 1997; DeWitt 1998; DeWitt et al. 1998; Scheiner and Berrigan 1998). Clonal plants are well-suited for such experiments because vegetative reproduction allows genotypes to be easily replicated and grown across different environ- 
ments. The chance of detecting costs and limits of plasticity is highest when there are significant genotype-by-environment interactions (DeWitt 1998; De Witt et al. 1998). However, even in the absence of such interactions, costs and limits of plasticity may be detected when a large number of genotypes is included in the analysis.

We investigated potential costs and limits of plastic responses in lengths of leaves and stolon internodes to competition using 102 genotypes from 14 populations of the clonal herb Ranunculus reptans. Competition is an ecologically relevant factor for $R$. reptans because this species grows on lake shores with a typical gradient from competition-free to competitive environments (Prati 1998). We grew three vegetatively propagated replicates of each genotype with and three without competition by the naturally most abundant cooccurring grass species, Agrostis stolonifera. We measured plasticity in lengths of leaves and stolon internodes and used multiple regression to determine whether more plastic genotypes had a lower fitness in terms of the production of rosettes, rooted rosettes, and flowers than less plastic genotypes with the same phenotype. We tested for developmental instability costs by calculating genetic correlations of plasticity with within-environment variation (SD). Additionally, we tested for a developmental range limit by correlation and regression analyses of trait plasticity on trait averages over both environments.

We asked the following specific questions. Does competition affect lengths of leaves and stolon internodes of $R$. reptans, is there variation among genotypes in these traits, and is there variation among genotypes in their plastic response to competition? Are there costs of plasticity in lengths of leaves and stolon internodes of $R$. reptans? Are more plastic genotypes developmentally less stable than less plastic genotypes? Is the developmental range narrower for more plastic genotypes than for less plastic genotypes? We discuss our methods and results in the light of the few other studies on costs and limits of plasticity.

\section{Materials And Methods}

\section{Study Species}

The stoloniferous, rosette-forming herb R. reptans L. (Ranunculaceae) has a circumpolar distribution, mainly in the temperate to boreal-subarctic zones of Europe, Asia, and North America (Hess et al. 1980). The species has a strict habitat requirement and in central Europe only grows on periodically inundated lake shores with low vegetation cover. Today, most populations are found around Lake Constance, which is located at the border of Austria, Germany, and Switzerland. Within these populations, there is a consistent gradient between microhabitats. Plants growing close to the winter water level of the lake experience an average summer-inundation period of 150 days and little competition with other species. In contrast, plants growing about $30 \mathrm{~cm}$ higher and $10 \mathrm{~m}$ further away from the lake experience an average summerinundation period of 80 days and competition with graminoids, most commonly Agrostis stolonifera (Prati 1998).

Rosettes of $R$. reptans may have up to 20 leaves. Leaf blades are $10-50 \mathrm{~mm}$ long and $1-5 \mathrm{~mm}$ wide and gradually narrow into the petioles. Rosettes form stolon branches from meristems in the axils of leaves. Stolons grow sympodially and consist of rooted or unrooted rosettes connected by thin (0.5-2.0 mm diameter) stolon internodes with a length of 3$5 \mathrm{~cm}$. Stolon branches can grow as long as 10-20 cm within one growing season. Each rosette may produce one single flower and one or more side branches (Prati and Peintinger 2000). The production of stolon branches is interrupted by the summer inundation period, during which stolon internodes decay (D. Prati, pers. comm.).

\section{Plant Material}

To sample potential genetic variation both within and among populations, we collected 104 plants from 14 populations around Lake Constance in 1995 and 1997. The area covered by $R$. reptans in these populations ranged from $1-10^{4}$ $\mathrm{m}^{2}$ (Fischer et al. 2000). Distances between sampled plants were $\geq 5 \mathrm{~m}$ in all cases and random amplified polymorphic DNA (RAPD) analysis revealed that the 104 plants represented 102 different genotypes (Fischer et al. 2000). Collected plants were propagated repeatedly, and vegetative offspring were kept in a plant room at $25^{\circ} \mathrm{C}$ with $16 \mathrm{~h}$ of artificial light in $40 \times 70 \mathrm{~cm}$ trays filled with a $4: 1$ mixture of sand and compost.

\section{Experimental Set-up}

For the experiment, which was performed in the same plant room as the precultivation, we filled $7831 \times 44 \mathrm{~cm}$ trays with a soil mixture of the same composition as during precultivation and covered it with a thin layer of gravel (to prevent growth of algae and to reduce evaporation). On 14 November 1997, we randomly assigned three rosettes of each of the 102 genotypes (two genotypes were represented by six rosettes) to the eight planting positions in each of 39 trays (total of 312 plants), with the limitation that rosettes planted to the same tray belonged to different genotypes. We did the same for the other 39 trays (also 312 plants) in which we additionally sowed A. stolonifera as competitor. Agrostis stolonifera formed a dense matrix with about one plant per $\mathrm{cm}^{2}$, which is comparable to densities found in the natural habitat of $R$. reptans at Lake Constance. Within trays, interference among $R$. reptans individuals is likely to be weak, because rooting zones of the originally planted rosettes did not overlap and because $R$. reptans plants impose hardly any shading. Plants were watered every second day, and trays were assigned to new random positions in the plant room weekly.

\section{Measurements}

Eight weeks after the start of the experiment, we measured the length of the longest leaf and the length of the first three internodes of the longest stolon branch on each originally planted rosette. The first three internodes of the stolon branches did not reach the border of the trays and did not interfere with other $R$. reptans individuals within the same tray. Eight weeks is representative of the duration of a natural inundation-controlled vegetation period of $R$. reptans at Lake Constance. For each plant developing from the originally planted rosettes, we counted the total number of rosettes, rooted rosettes, and flowers as measures of fitness. 


\section{Statistical Analysis}

We tested the effects of competition, variation among trays, variation among populations of origin, and variation among genotypes with analyses of variance (using the statistical software SPSS, SPSS, Inc., Chicago). We considered competition as a fixed factor and tray, population and genotype as random factors. The factor tray was nested within competition, and the factor genotype was nested within population.

On the one hand, elongation of leaves and internodes in response to shade imposed by competing plants is generally thought to be adaptive, and thus to be an active form of plasticity (Smith 1982; Schmitt and Wulff 1993; Schmitt et al. 1995; van Hinsberg 1996; Schlichting and Pigliucci 1998). On the other hand, reduced availability of resources under competition may reduce lengths of leaves and internodes, and may thus result in a passive form of plasticity. Costs of plasticity are only expected for active plasticity. Therefore, we consider genotypes that respond to competition by elongation of leaves and internodes as most plastic, genotypes that maintain the same lengths as intermediately plastic, and genotypes that have reduced lengths of leaves and internodes under competition as least plastic. Consequently, we calculated plasticity by subtracting the trait value in the competition free environment from the trait value in the competitive environment.

Costs of plasticity. - In a focal environment, a cost of plasticity is present when a more plastic genotype exhibits lower fitness in this focal environment than a less plastic genotype with the same trait value (DeWitt et al. 1998). In both competition environments $(k)$, we tested for costs of plasticity by regression of the mean fitness of a genotype $(j)$ in the focal environment $\left(\bar{W}_{j, k}\right)$ on the genotypic mean trait value in the focal environment $\left(\bar{Z}_{j, k}\right)$ and the genotypic mean plasticity over both environments $\left(\bar{P}_{j}=\bar{Z}_{j, k=2}-\bar{Z}_{j, k=1}\right.$; eq. 1 ; van Tienderen 1991; Scheiner and Berrigan 1998):

$$
\bar{W}_{j, k}=\text { constant }_{k}+\alpha_{k} \overline{\mathrm{Z}}_{j, k}+\beta_{k} \bar{P}_{j} \text {. }
$$

Because only a significantly negative regression coefficient of fitness on plasticity (i.e., a selection gradient) indicates costs of plasticity, we used one-tailed significance tests. To allow comparisons between coefficients, we standardized regression coefficients (i.e., we expressed them in units of standard deviations).

Developmental instability costs. - There are two prerequisites before plasticity can be considered to be associated with developmental instability costs. First, plasticity in a trait should be positively correlated with developmental instability of this trait. Second, developmental instability in this trait should be negatively correlated with fitness.

Developmental instability of a genotype can be measured as the within-environment variation among its replicates. By this definition, we imply that such within-environment variation is generally not adaptive and does not represent plasticity of another kind (Schmid 1992). To test whether more plastic genotypes are developmentally less stable than less plastic genotypes, we calculated genetic correlations between within-environment variation of a trait, measured as standard deviations (SDs), and trait plasticity (Scheiner et al. 1991;
DeWitt 1998). Because only positive correlations indicate that this first prerequisite for developmental instability costs is fulfilled, we used one-tailed significance tests.

The calculation of within-environment SD of a trait is mathematically not independent from trait plasticity calculated from the same data. A correlation coefficient calculated between within-environment SD and plasticity could give a positive or negative correlation even in the absence of a biological relationship between the two parameters (i.e., spurious correlation). We applied a randomization procedure to test if there was a biological relationship in our study that exceeds the one that can be expected as a consequence of a spurious correlation (Edgington 1986). We randomized all observations with regard to genotypes within a focal environment 1000 times. Each time we calculated within-environment SD of a trait and trait plasticity, and the correlation between these two parameters (using the statistical software GENSTAT, Lawes Agricultural Trust, IACR, Rothamsted). The distribution of the resulting 1000 correlation coefficients was used for a test of significance of the correlation between both parameters, and the mean of this distribution served as estimate of the spurious correlation.

Developmental range limit.-The developmental range might be narrower in more plastic genotypes than in less plastic genotypes. In the case that there are only extremes with high trait values, such a limit of plasticity would be noticed as a negative correlation between the trait averages of genotypes over both environments $\left(\left[\bar{Z}_{j, k=1}+\bar{Z}_{j, k=2}\right] / 2\right)$ and trait plasticity $\left(\bar{Z}_{j, k=2}-\bar{Z}_{j, k=1}\right)$; Fig. 1). In the case that there are not only extremes with high trait values but also extremes with low trait values, such a limit of plasticity would be noticed as a concave-down relationship between both parameters (Fig. 1). To test these predictions, we calculated correlations between trait plasticity and trait averages over both environments and second-order polynomial regressions of trait plasticity on trait averages. A concave-down relationship would be indicated by a negative regression coefficient of the quadratic term.

To correct for spurious correlations between trait plasticity and trait averages over both environments, we used a randomization procedure (Edgington 1986) similar to the one described above. We randomized trait mean values of genotypes in one environment with regard to trait mean values in the other environment 1000 times. Each time, we calculated trait plasticity, trait averages over both environments, the correlation between both parameters, and the $t$-values of the coefficients of the second-order polynomial regression of plasticity on trait averages (using the statistical software GENSTAT). The distribution of the resulting 1000 correlation coefficients was used for a test of significance of the correlation between both parameters, and the mean of this distribution served as estimate of the spurious correlation. However, the significance of the 1000 regression coefficients in the distribution is not determined by the coefficients themselves, but by their $t$-values (i.e., regression coefficients divided by their standard errors). Therefore, in contrast to the significance tests of correlations, we used the distribution of $t$-values for tests of significance of coefficients of the quadratic term in the second-order polynomial regression. Because we had explicit expectations for the signs of the cor- 
A

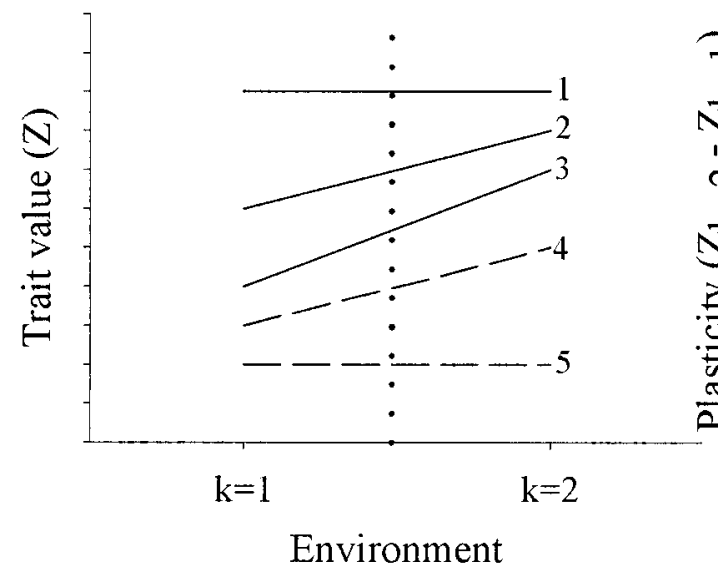

B

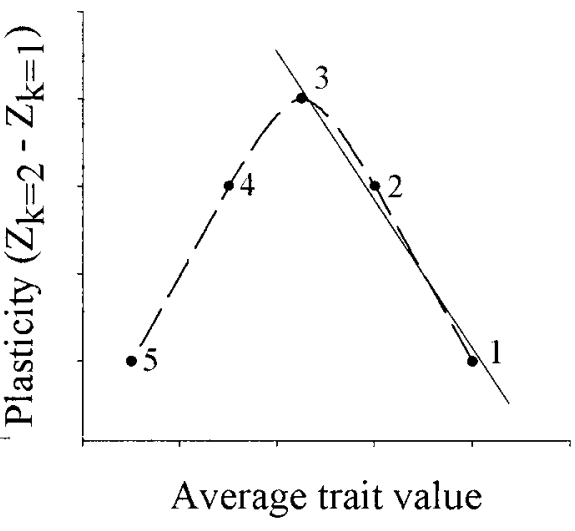

FIG. 1. Graphical illustration of the hypothesis of a narrower developmental range for more plastic genotypes compared with less plastic genotypes in the case of adaptive plasticity (i.e., high trait values are beneficial in environment $k=2$ ). The plastic genotype 3 has a lower trait average than the less plastic genotype 2 and the nonplastic genotype 1 (solid lines in A). This lowering of the upper developmental limit would correspond to a negative relation, shown by the solid line in B, between the trait average over both environments (which lies on the dotted line in A) and trait plasticity. When the lower extreme values are only possible for less plastic or nonplastic genotypes (4 and 5), the relation between trait averages and trait plasticity will be concave-down (dashed line in B).

relation and regression coefficients, we used one-tailed significance tests.

\section{RESULTS}

After eight weeks, $R$. reptans plants grown under competition had about half as many rosettes $(42 \%)$, rooted rosettes $(60 \%)$, and flowers $(39 \%)$ than those grown without competion (Table 1). Plant mortality during the experiment was $5.4 \%$ under competition and $0.6 \%$ without competition. Under competition originally planted rosettes had grown both shorter leaves $(-14 \%)$ and stolon internodes $(-6 \%)$ than without competition (Table 1). Genetic correlations between pairs of fitness measures and between the two foraging traits were positive $(r>0.57, P<0.001$ in all cases). For all these traits, both the effect of competition and variation among genotypes were significant (Table 2A,B). The plasticity in leaf length (mean $\pm \mathrm{SE}=-5.2 \pm 0.7$ ) ranged from -32.7 to 21.0 and differed significantly among genotypes from different populations (competition-by-population interaction term in Table $2 \mathrm{~B}$ ). The plasticity in length of the first three

TABLE 1. The effect of competition on the mean ( \pm SE) of fitness measures (number of rosettes, rooted rosettes, and flowers) and foraging traits (length of longest leaf and first three stolon internodes of originally planted rosettes) in the experiment with Ranunculus reptans. Experimental plants represented 102 genotypes from 14 natural populations.

\begin{tabular}{lrc}
\hline \hline \multicolumn{1}{c}{ Trait } & $\begin{array}{c}\text { Absence of } \\
\text { competition }\end{array}$ & $\begin{array}{c}\text { Presence of } \\
\text { competition }\end{array}$ \\
\hline Fitness measures & $19.3 \pm 0.7$ & $8.2 \pm 0.3$ \\
$\quad$ Number of rosettes & $5.0 \pm 0.2$ & $3.0 \pm 0.1$ \\
$\quad$ Number of rooted rosettes & $8.0 \pm 0.3$ & $3.1 \pm 0.2$ \\
$\quad$ Number of flowers & $34.7 \pm 0.5$ & $29.9 \pm 0.6$ \\
$\begin{array}{l}\text { Foraging traits } \\
\quad \text { Length of the longest leaf }(\mathrm{mm})\end{array}$ & $78.3 \pm 1.2$ & $73.6 \pm 1.4$ \\
$\quad \begin{array}{l}\text { Length of first three internodes } \\
(\mathrm{mm})\end{array}$ & & \\
\hline
\end{tabular}

stolon internodes (mean $\pm \mathrm{SE}=-4.8 \pm 1.5$ ) ranged from -42.7 to 41.7 . The variation in plasticity among genotypes, however, was not significant (Table 2). The genetic correlation between plasticity in leaf length and plasticity in internode length was weak and not significant $(r=-0.033, P$ $=0.746)$.

\section{Costs of Plasticity}

Regression coefficients indicating the influence of mean values of foraging traits on fitness measures in the focal environment were positive and in most cases significant, whether the focal environment was with or without competition (Table 3). This suggests that selection favors plants with long leaves and stolon internodes in both environments and thus that long leaves and stolon internodes indeed are adaptive in $R$. reptans.

In the competitive focal environment all regression coefficients indicating the influence of trait plasticities on fitness measures were negative (Table 3 ). The coefficients were significant for internode length when fitness was measured in terms of the number of rosettes (selection gradient -0.250 ; Fig. 2, Table 3) and the number of flowers (selection gradient -0.214 ; Table 3 ). In other words, more plastic genotypes with the same internode length as less plastic genotypes in the presence of competition produced fewer rosettes and flowers than the less plastic genotypes in this focal environment. This indicates that there are costs of plasticity in internode length in the presence of competition. In the competition-free focal environment regression coefficients indicating the influence of trait plasticities on fitness measures were all close to zero and not significant (Table 3 ). This suggests that genotypes did not incur costs of plasticity in the absence of competition.

\section{Developmental Instability Costs}

Genetic correlations between developmental instability in a trait and trait plasticity were negative for all traits (Fig. 3; 
TABLE 2. Summary of analyses of variance of effects of competition on and of variation among trays, among populations of origin, and among genotypes in (A) fitness measures (number of rosettes, rooted rosettes, and flowers) and (B) foraging traits (length of longest leaf and first three stolon internodes of the originally planted rosettes) in the experiment with Ranunculus reptans. Experimental plants represented 102 genotypes from 14 natural populations. C, competition; P, population; G, genotype.

\begin{tabular}{|c|c|c|c|c|c|c|c|}
\hline \multirow{2}{*}{ A Effect } & \multirow[b]{2}{*}{ df } & \multicolumn{2}{|c|}{ Number of rosettes } & \multicolumn{2}{|c|}{ Number of rooted rosettes } & \multicolumn{2}{|c|}{ Number of flowers } \\
\hline & & MS & $F$ & MS & $F$ & MS & $F$ \\
\hline Competition & 1 & 18583.86 & $134.93 * * *$ & 629.35 & $106.07 * * *$ & 3475.03 & $115.45^{* * *}$ \\
\hline $\operatorname{Tray}(\mathrm{C})$ & 76 & 137.70 & 2.53 & 5.93 & $1.35 *$ & 30.10 & $2.02 * * *$ \\
\hline Genotype(P) & 88 & 112.27 & $2.06^{* * *}$ & 5.88 & $1.34 *$ & 31.93 & $2.14 * * *$ \\
\hline $\mathrm{C} \times \mathrm{P}$ & 13 & 63.75 & 0.91 & 3.24 & 0.85 & 19.44 & 1.30 \\
\hline $\mathrm{C} \times \mathrm{G}(\mathrm{P})$ & 88 & 69.95 & 1.28 & 3.79 & 0.80 & 21.62 & $1.45^{*}$ \\
\hline Effect & df & & & $F$ & $\mathrm{df}$ & MS & $F$ \\
\hline Competition & 1 & 37 & & $33.60 * * *$ & 1 & 2591.71 & $7.09 * *$ \\
\hline $\operatorname{Tray}(\mathrm{C})$ & 76 & & & $2.17 * * *$ & 75 & 365.63 & $1.50 *$ \\
\hline Population & 13 & & & 1.58 & 13 & 1090.32 & 1.49 \\
\hline Genotype(P) & 88 & & & $3.60 * * *$ & 87 & 731.66 & $3.00 * * *$ \\
\hline $\mathrm{C} \times \mathrm{P}$ & 13 & & & $2.03 *$ & 13 & 176.93 & 0.80 \\
\hline
\end{tabular}

$\dagger P<0.1 ; * P<0.05 ; * * P<0.01 ; * * * P<0.001$.

Table 4). Correspondingly, none of the coefficients was significant with one-tailed significance tests (Table 4). This indicates that more plastic genotypes were developmentally not less stable than less plastic genotypes. On the contrary, more plastic genotypes were even developmentally more stable than less plastic ones, as indicated by the significance of correlations when we used two-tailed tests. Because these results already exclude the possibility of developmental instability costs, it was not necessary to also test whether developmental instability was negatively correlated with fitness.

\section{Developmental Range Limit}

Correlations between trait plasticities and trait averages over both environments were positive (Fig. 4). Correspondingly, they were not significant when we used one-tailed significance tests, however, they were significant when we used two-tailed tests $(P<0.001$; Table 5$)$. This indicates that more plastic genotypes were not less likely than less plastic genotypes to produce extremely long leaves or stolon internodes, but, rather, that they were more likely to do so.

Second-order polynomial regressions of trait plasticities on trait averages yielded positive coefficients for the quadratic terms $\left([Z]^{2}\right.$ in Table 5). Although coefficients were not significant (Table 5), the results nevertheless suggest that the relationship was not concave-down but rather concave-up. Therefore, less plastic genotypes do not appear to have a greater potential to produce extreme trait values than more plastic genotypes.

\section{DISCUSSION}

\section{Methodological Considerations}

Phenotypic costs of plasticity consist of both environmental and genetic effects. An example for phenotypic production costs is reduced fitness observed after experimental induction

TABLE 3. Summary of regression analyses of fitness measures on both mean value and plasticity in foraging traits of Ranunculus reptans according to equation (1). Here mean values are genotypic trait values in a focal environment with or without competition $\left(Z_{j k=1}\right.$ or $\left.Z_{j k=2}\right)$, and plasticities are genotypic trait plasticities $\left(P_{j}\right)$. Experimental plants represented 102 genotypes from 14 natural populations. Foraging traits are length of the longest leaf and length of first three stolon internodes of the originally planted rosettes. Fitness was measured as number of rosettes, rooted rosettes, and flowers. Costs of plasticity are indicated by negative regression coefficients of $\bar{P}_{j}$.

\begin{tabular}{|c|c|c|c|c|}
\hline \multirow{2}{*}{$\begin{array}{l}\text { Fitness measure (in italics) } \\
\text { and foraging trait }\end{array}$} & \multicolumn{2}{|c|}{ Absence of competition } & \multicolumn{2}{|c|}{ Presence of competition } \\
\hline & $\bar{Z}_{j k=1}$ & $\bar{P}_{j}$ & $\bar{Z}_{j k=2}$ & $\bar{P}_{j}$ \\
\hline \multicolumn{5}{|l|}{ Number of rosettes } \\
\hline Length of the longest leaf & $0.321 * *$ & 0.081 & $0.432 * * *$ & -0.107 \\
\hline Length of first three internodes & $0.398 * * *$ & -0.038 & $0.556 * * *$ & $-0.250 *$ \\
\hline \multicolumn{5}{|l|}{ Number of rooted rosettes } \\
\hline Length of the longest leaf & 0.117 & 0.094 & $0.354 * *$ & -0.156 \\
\hline Length of first three internodes & $0.340 * * *$ & 0.005 & $0.470 * * *$ & -0.128 \\
\hline \multicolumn{5}{|l|}{ Number of flower } \\
\hline Length of the longest leaf & $0.292 * *$ & 0.099 & $0.395 * * *$ & -0.083 \\
\hline Length of first three internodes & $0.337 * * *$ & -0.082 & $0.529 * * *$ & $-0.214^{*}$ \\
\hline
\end{tabular}

$* P<0.05 ; * * P<0.01 ; * * * P<0.001$ 


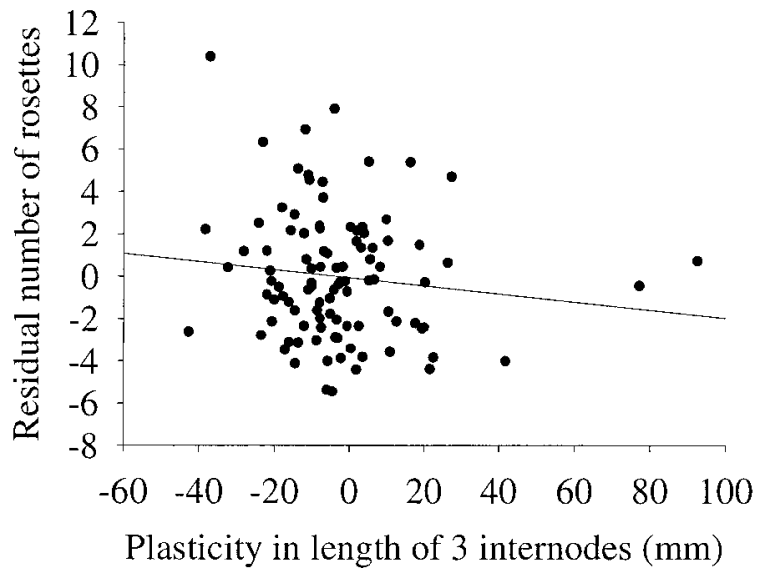

FIG. 2. Illustration of costs of plasticity in internode length ( $\mathrm{mm})$ of Ranunculus reptans in response to competiton. The relationship between residual number of rosettes under competition and plasticity in length of the first three stolon internodes of the originally planted rosette is significant at the 5\% level $(r=-0.205, P<$ $0.05)$. The residual number of rosettes was obtained from the regression of the number of rosettes in the presence of competition on the length of the first three stolon internodes in the presence of competition. Experimental plants represented 102 genotypes from 14 natural populations.

of biochemical plant defense to herbivory (Brown 1988, Baldwin 1998). Costs of producing a structure may depend on the environment in which the structure is produced, and they may differ among genotypes. For the evolution of plasticity, however, only those costs of plasticity that are genetic in origin are relevant (Agrawal et al. 1999). We explicitly investigated potential genetic costs and limits of plasticity because we used genotypic values in all our regression and correlation analyses.

Although the range of plasticities were large for both leaf and internode length of $R$. reptans, we only found significant variation among genotypes from different populations of origin in their plastic leaf length response to competition (Table 2B). Nevertheless, the large range of plasticities and the high number of genotypes used in our study gave our regression analyses enough power to detect costs of plasticity in internode length.

Analysis of costs of plasticity.-The analysis of costs of plasticity according to equation (1) is a selection gradient analysis (Lande and Arnold 1983) and was first brought up by van Tienderen (1991) and later adapted for the use of genotypic trait values by Scheiner and Berrigan (1998).

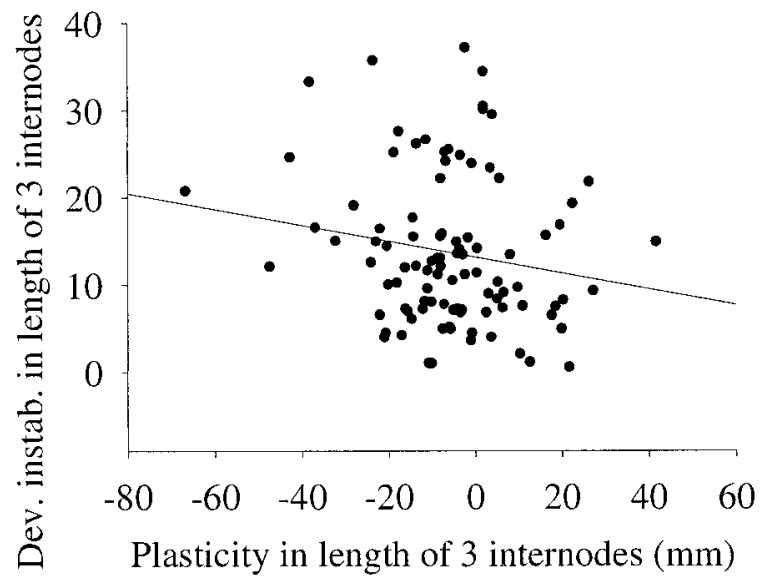

FIG. 3. Relationship between developmental instability in the length $(\mathrm{mm})$ of the first three stolon internodes of originally planted rosettes of Ranunculus reptans grown without competition and plasticity in this trait in response to competition. Developmental instability was measured as standard deviation among replicates per genotype. Experimental plants represented 102 genotypes from 14 natural populations. A positive correlation between both parameters would be a prerequisite for developmental instability costs. In contrast, the negative correlation between both parameters was significant (two-tailed test, $r=-0.168, P<0.05$; see Table 4).

DeWitt (1998) and Scheiner and Berrigan (1998) proposed to add interaction and quadratic terms to this regression model. The interaction term between trait value in the focal environment and plasticity $\left(Z_{j, k} P_{j}\right)$ would reveal whether costs of plasticity vary with the genotypic trait value in the focal environment (DeWitt 1998; Scheiner and Berrigan 1998). Quadratic terms would give information on whether there are nonlinear components of selection (i.e., stabilizing or disruptive selection; Lande and Arnold 1983; Scheiner and Berrigan 1998). However, addition of interaction and quadratic terms to the model has the disadvantage that estimates of the regression coefficients may be less reliable as a consequence of high correlations between the independent variables (i.e., multicollinearity; Mitchell-Olds and Shaw 1987; Jobson 1991) and that the explanatory model becomes less parsimonious. Therefore, we did not add such terms to our regression model.

Spurious correlations. - Analyses of developmental instability costs and of the developmental range limit are based on correlations (and second-order polynomial regression) between trait plasticity and within-environment variation (SD) of the trait or the trait average, respectively. Use of the same

TABLE 4. Correlations coefficients between developmental instability and plasticity in response to competition in two foraging traits in Ranunculus reptans. Traits are length of longest leaf and length of first three stolon internodes of originally planted rosettes. Experimental plants represented 102 genotypes from 14 natural populations. Developmental instability of a genotype was measured as the standard deviation of the trait in a focal environment. Developmental instability costs would be indicated when the observed correlation coefficients are positive (i.e., larger than the effect due to spurious correlations). We used a randomization procedure to estimate the effect due to spurious correlations and to test for (one-tailed) significance of the observed coefficients. The observed correlations coefficients are not significant because they are not larger than the $95 \%$ quantile.

\begin{tabular}{|c|c|c|c|c|c|c|}
\hline \multirow[b]{2}{*}{ Foraging trait } & \multicolumn{3}{|c|}{ Absence of competition } & \multicolumn{3}{|c|}{ Presence of competition } \\
\hline & Observed & Spurious & $95 \%$ quantile & Observed & Spurious & $95 \%$ quantile \\
\hline Length of longest leaf & -0.334 & -0.124 & 0.032 & -0.016 & 0.086 & 0.232 \\
\hline Length of first three internodes & -0.168 & 0.007 & 0.165 & -0.033 & 0.047 & 0.197 \\
\hline
\end{tabular}


A

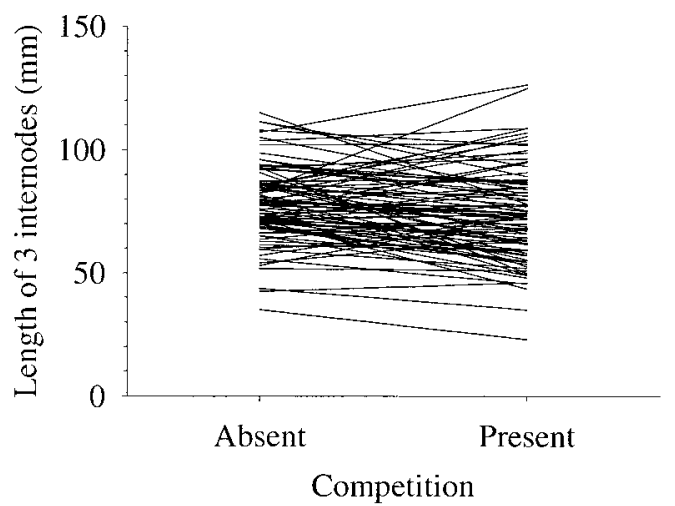

$\mathrm{B}$

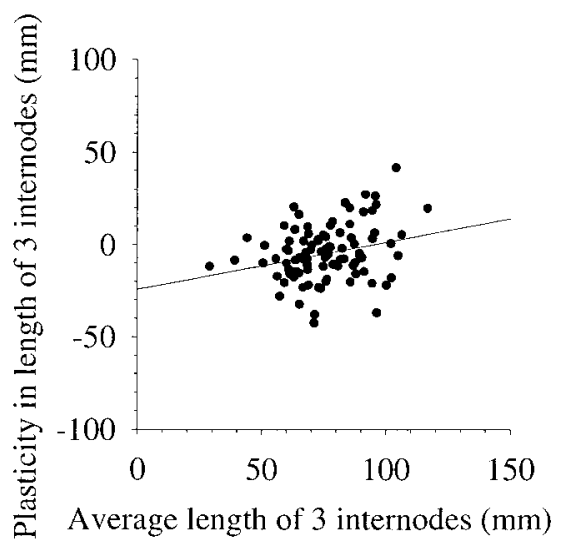

FIG. 4. Developmental range of internode lengths of Ranunculus reptans (see Fig. 1). (A) Response to competition of the length of the first three stolon internodes of the originally planted rosettes of 98 genotypes. (B) Relationship between trait plasticity and averages of the length of the first three stolon internodes over both competition environments. Experimental plants represented 102 genotypes from 14 natural populations. A negative relationship would indicate a developmental range limit. In contrast, the positive correlation between plasticity in the length of the first three stolon internodes and the average of this trait over both environments was significant (two-tailed test, $r=0.266, P<0.001$; see Table 5).

replicates in the estimates of both parameters may confound the results with spurious correlations. Therefore, we used randomization procedures to estimate the effects of spurious correlations and to test the significance of the results. Especially in the tests of the developmental range limit, the effects of spurious correlations turned out to be large (Table 5 ), indicating that conclusions without consideration of the spurious nature of the correlation may be erroneous (DeWitt 1998).

To avoid problems caused by spurious correlations, an alternative method would be the use of one set of replicates for the estimation of plasticity and of another for estimation of the other two parameters. Although such a procedure avoids wrong conclusions based on mathematical artifacts altogether, it reduces the power of the analysis. Application of this procedure to our data did not change our conclusions (data not shown). We strongly recommend either applying randomization procedures or using different replicates to calculate parameters, whenever parameters are mathematically not independent from each other.

Analysis of a developmental range limit.-To test for a developmental range limit, we used both correlation analysis and second-order polynomial regression (see Material and Methods, Fig. 1). DeWitt (1998) used another method in which he calculated correlations between the trait value in a focal environment and plasticity in that trait. In the environment selecting the trait value, he expected negative correlations between both parameters because less plastic genotypes would have higher trait values (upper extremes) when compared to more plastic genotypes (environment $k=1$ in Fig. 1, not considering genotypes 4 and 5). In the other environment, he expected positive correlations between both parameters because less plastic genotypes would have lower trait values (lower extremes) when compared to more plastic genotypes. However, in both environments genotypes of low plasticity may occur with extremely high trait values and others with extremely low trait values (consider all genotypes in Fig. 1). Consequently the signs of the correlations between plasticity and the trait value in a focal environment may be opposite to the ones expected by DeWitt (1998). For example, in Figure 1 the correlation between plasticity and trait values in environment $k=1$ is not positive but slightly negative,

TABLE 5. Tests for a developmental range limit to the plasticity in foraging traits of Ranunculus reptans by correlations between trait plasticities and trait averages over two competition environments and by second-order polynomial regression analyses of trait plasticities on trait averages. Foraging traits were length of longest leaf and length of first three stolon internodes of the originally planted rosettes grown in the absence or presence of competition. Experimental plants represented 102 genotypes from 14 natural populations. A developmental range limit would lead to either a negative correlation or a negative regression coefficient of $(Z)^{2}$ in the second-order polynomial regression (i.e., smaller than the effect due to spurious correlations). We used randomization procedures to estimate the effect due to spurious correlations and to test for (one-tailed) significance of the observed coefficients. The observed correlation and regression coefficients are not significant because observed correlation coefficients and observed $t$-values are not smaller than the $5 \%$ quantile.

\begin{tabular}{|c|c|c|c|c|c|c|c|}
\hline \multirow[b]{3}{*}{ Trait } & \multirow{2}{*}{\multicolumn{3}{|c|}{ Correlation }} & \multicolumn{4}{|c|}{ Second-order polynomial regression } \\
\hline & & & & \multirow[b]{2}{*}{$(\bar{Z})^{2}$} & \multicolumn{2}{|c|}{$t$-values } & \multirow[b]{2}{*}{$5 \%$ quantile } \\
\hline & Observed & Spurious & $5 \%$ quantile & & Observed & Spurious & \\
\hline Length of first three internodes & 0.266 & 0.197 & 0.144 & 0.871 & 1.34 & 0.96 & -0.54 \\
\hline
\end{tabular}


whereas the correlation between plasticity and trait values in environment $k=2$ is not negative but slightly positive. In fact, in his study on predator-induced plasticity in the freshwater snail P. heterostropha, DeWitt (1998) reported significant correlations between trait values and plasticity with signs opposite to the ones that he had expected.

\section{Costs of Plasticity}

Type of costs of plasticity detected in Ranunculus reptans.Genotypes of $R$. reptans that were more plastic in the length of stolon internodes produced fewer rosettes and flowers in the presence of competition than less plastic genotypes when they had the same internode length in this focal environment. The detection of costs of plasticity itself, however, does not directly reveal the nature of these costs.

In the case of $R$. reptans, developmental instability costs can be excluded, because plasticity was not negatively associated with developmental instability. In his study on predator-induced plasticity in the freshwater snail $P$. heterostropha, DeWitt (1998) did not detect developmental instability costs either. Scheiner et al. (1991) report significantly positive correlations between developmental instability and plasticity, but only for some traits and for some temperature environments in Drosophila melanogaster. The results of these two studies, however, may be compromised by spurious correlations, because the same data were used in estimates of within-environment variation and of trait plasticity.

Costs of information acquisition are probably not important in plants, which in contrast to most animals sample their environment less actively. Production costs are not very likely in our study, because there is no obvious reason why the production costs of stolon internodes should be higher for more plastic than for less plastic genotypes. Energetic costs of maintenance of the genetic and physiological machinery necessary for plasticity should be present in both environments (Scheiner and Berrigan 1998). The absence of costs of plasticity in the absence of competition may therefore suggest that the costs of plasticity do not represent maintenance costs. However, although maintenance costs should be equal in both environments in an absolute sense, they will be smaller in a relative sense in the less stressful environment. Thus, maintenance costs will be more difficult to detect in benign environments than in stressful environments. Indeed, in the absence of competition, regression coefficients of fitness on plasticity were negative, although non-significantly, only for the two combinations of fitness measure and foraging trait that revealed significant costs of plasticity in the presence of competition (Table 3). Therefore, we suggest that detected costs of plasticity in internode length of $R$. reptans are costs of maintenance of the developmental and physiological machinery necessary for a plastic response.

Studies on costs of plasticity. - Several theoretical studies have shown that costs of plasticity may constrain the evolution of plasticity (van Tienderen 1991, 1997; Moran 1992; León 1993; Padilla and Adolph 1996). However, although badly needed to better understand the evolution of plasticity, empirical studies on costs of plasticity are very scarce (Via et al. 1995).

An empirical study on costs of plasticity to predator cues in the freshwater snail $P$. heterostropha reported evidence for a cost of plasticity in a morphological trait in only one of nine analyses (DeWitt 1998). Scheiner and Berrigan (1998) report that there was no evidence for costs of plasticity in any of three morphological traits of Daphnia pulex kept in the absence or presence of predator cues. The only study on costs of plasticity in plants reports costs of plasticity in leaf thickness, one of the seven physiological and morphological traits studied in Plantago coronopus grown with or without salinity stress (Smekens 1998). So, in the few cases where costs of plasticity have been sought, they have been found only for a very limited number of traits.

In principle, this may either indicate that costs of plasticity are absent or that costs of plasticity are difficult to detect. Detection of costs of plasticity may be difficult because genetic variation for plasticity is generally low (Scheiner 1993; DeWitt 1998). However, even in the absence of significant genotype-by-environment interactions, costs of plasticity may be detected (plasticity in internode length in this study). Furthermore, plasticity is trait-specific and environment-specific (Bradshaw 1965). Plasticity in leaf length and plasticity in internode length of $R$. reptans were not correlated in our study. However, in principle, genotypes that are more plastic in one trait or more plastic in response to one environmental factor may be less plastic for another trait or less plastic in response to other environmental factors. This implies that detection of plasticity costs might be obscured by negative correlations between plasticity costs in different traits or by negative correlations between plasticity costs to different environmental factors. Such patterns might be unraveled in more elaborate experiments with multiple traits and multiple environments.

\section{Constraints on the Evolution of Foraging in Stoloniferous Plants}

We found evidence for costs of plasticity in internode length, while there was no evidence for costs of plasticity in leaf length. Therefore, even when there are undetected costs of plasticity in leaf length, they are likely to be smaller than costs of plasticity in internode length. This may suggest that selection has acted more strongly against costs of plasticity in leaf length than against those in internode length of $R$. reptans. However, selection is expected to act more strongly against costs of nonadaptive plasticity than against costs of adaptive plasticity (DeWitt 1998). Because the light climate in natural vegetation is less predictable in the horizontal plane than in the vertical plane, plasticity in internode length of stoloniferous plants may be less adaptive than plasticity in leaf length (Huber 1996; Huber et al. 1998). Therefore, stronger selection against costs of plasticity in leaf length than in internode length is not a very likely explanation of higher costs of the latter. In general, stoloniferous plants show less plasticity in internode length than in leaf length (de Kroon and Hutchings 1995; Huber 1996; Huber et al. 1998). Therefore, we conclude that costs of plasticity may constrain the evolution of foraging in the horizontal plane (i.e., internode length) more strongly than in the vertical plane (i.e., leaf length) in $R$. reptans and possibly in stoloniferous plants in general. 


\section{ACKNOWLEDGMENTS}

We thank T. Pfluger, R. Schmid, and A. Tchouboukov for practical assistance, M. Kéry for help with the randomization procedures, P. van Tienderen for a stimulating discussion, and S. Scheiner and an anonymous reviewer for comments on an earlier draft. We acknowledge financial support by the Swiss National Science Foundation (grant 31-49728.96).

\section{Literature Cited}

Agrawal, A. A., S. Y. Strauss, and M. J. Stout. 1999. Costs of induced responses and tolerance to herbivory in male and female fitness components of wild radish. Evolution 53:1093-1104.

Baldwin, I. T. 1998. Jasmonate-induced responses are costly but benefit plants under attack in native populations. Proc. Natl. Acad. Sc. USA 95:8113-8118.

Bradshaw, A. D. 1965. Evolutionary significance of phenotypic plasticity in plants. Adv. Genet. 13:115-155.

Brown, D. G. 1988. The cost of plant defense: an experimental analysis with inducible proteinase inhibitors in tomato. Oecologia 76:467-470.

de Kroon, H., and M. J. Hutchings. 1995. Morphological plasticity in clonal plants: the foraging concept reconsidered. J. Ecol. 83: $143-152$.

DeWitt, T. J. 1998. Costs and limits of phenotypic plasticity: tests with predator-induced morphology and life history in a freshwater snail. J. Evol. Biol. 11:465-480.

DeWitt, T. J., A. Sih, and D. S. Wilson. 1998. Costs and limits of phenotypic plasticity. Trends Ecol. Evol. 13:77-81.

Edginton, E. S. 1986. Randomization tests. Marcel Dekker, New York.

Fischer, M., R. Husi, D. Prati, M. Peintinger, M. van Kleunen, and B. Schmid. 2000. RAPD variation among and within small and large populations of the rare clonal plant Ranunculus reptans (Ranunculaceae). Am. J. Bot. 87:1128-1137.

Harper, J. L. 1981. The concept of populations in modular organisms. Pp. 53-77 in R. M. May, ed. Theorertical ecology: principles and applications. 2d edition. Blackwell Scientific, Oxford, U.K.

Hess, H. E., E. Landolt, and R. Hirzel. 1980. Flora der Schweiz, Vol. 2. Birkhäuser, Basel, Switzerland.

Huber, H. 1996. Plasticity of internodes and petioles in prostrate and erect Potentilla species. Functional Ecology 10:401-409.

Huber, H., A. Fijan, and H. J. During. 1998. A comparative study of spacer plasticity in erect and stoloniferous herbs. Oikos 81: 576-586.

Hutchings, M. J., and H. de Kroon. 1994. Foraging in plants: the role of morphological plasticity in resource acquisition. Adv. Ecol. Res. 25:159-238.

Jobson, J. D. 1991. Applied multivariate data analysis. Vol. I. Regression and experimental design. Springer, New York.

Lande, R., and S. J. Arnold. 1983. The measurement of selection on correlated characters. Evolution 37:1210-1226.

León, J. A. 1993. Plasticity in fluctuating environments. Pp. 105121 in J. Yoshimura and C. W. Clark, eds. Adaptation in stochastic environments. Springer, Berlin.

Mitchell-Olds, T., and R. G. Shaw. 1987. Regression analysis of natural selection: statistical inference and biological interpretation. Evolution 41:1149-1161.

Møller A. P. 1997. Developmental stability and fitness: a review. Am. Nat. 149:916-932.

Moran, N. A. 1992. The evolutionary maintenance of alternative phenotypes. Am. Nat. 139:971-989.

Padilla, D. K., and S. C. Adolph. 1996. Plastic inducible morphogenesis are not always adaptive: the importance of time delays in a stochastic environment. Evol. Ecol. 10:105-117.

Pitelka, L. F., and J. W. Ashmun. 1985. Physiology and integration of ramets in clonal plants. Pp. 399-435 in J. B. C. Jackson, L. W. Buss, and R. E. Cook, eds. Population biology and evolution of clonal organisms. Yale Univ. Press, New Haven, CT.

Prati, D. 1998. The genetics and life-history evolution of the clonal plant Ranunculus reptans. Ph.D. diss., University of Zürich, Zürich.

Prati, D., and M. Peintinger. 2000. Biological flora of central Europe: Ranunculus reptans L. Flora 195:1128-1137.

Scheiner, S. M. 1993. Genetics and evolution of phenotypic plasticity. Annu. Rev. Ecol. Syst. 24:35-68.

Scheiner, S. M., and D. Berrigan. 1998. The genetics of phenotypic plasticity. VIII. The cost of plasticity in Daphnia pulex. Evolution 52:368-378.

Scheiner, S. M., R. L. Caplan, and R. F. Lyman. 1991. The genetics of phenotypic plasticity. III. Genetic correlations and fluctuating asymmetries. J. Evol. Biol. 4:51-68.

Schlichting, C. D., and M. Pigliucci. 1998. Phenotypic evolution: a reaction norm perspective. Sinauer Associates, Inc., Sunderland, MA.

Schmid, B. 1992. Phenotypic variation in plants. Evol. Trends Plants $6: 45-60$.

Schmitt, J., and R. D. Wulff. 1993. Light spectral quality, phytochrome and plant competition. Trends Ecol. Evol. 8:47-51.

Schmitt, J., A. C. McCormac, and H. Smith. 1995. A test of the adaptive plasticity hypothesis using transgenic and mutant plants disabled in phytochrome-mediated elongation responses to neighbors. Am. Nat. 146:937-953.

Smekens, M. 1998. On phenotypic plasticity in relation to salt tolerance in Plantago coronopus. Ph.D. diss., University of Utrecht, Utrecht, The Netherlands.

Smith, H. 1982. Light quality, photoperception and plant strategy. Annu. Rev. Plant Physiol. 33:481-518.

van Hinsberg, A. 1996. On phenotypic plasticity in Plantago lanceolata: light quality and plant morphology. Ph.D. diss., University of Utrecht, Utrecht, The Netherlands.

van Kleunen, M., M. Fischer, and B. Schmid. 2000. Clonal integration in Ranunculus reptans: by-product or adaptation? J. Evol. Biol. 13:237-248.

van Tienderen, P. H. 1991. Evolution of generalists and specialists in spatially heterogeneous environments. Evolution 45 1317-1331.

1997. Generalists, specialists, and the evolution of phenotypic plasticity in sympatric populations of distinct species. Evolution 51:1372-1380.

Via, S., R. Gomulkiewicz, G. de Jong, S. M. Scheiner, C. D. Schlichting, and P. H. van Tienderen. 1995. Adaptive phenotypic plasticity: consensus and controversy. Trends Ecol. Evol. 10: 212-217.

Wilson, D. S., and J. Yoshimura. 1994. On the coexistence of specialists and generalists. Am. Nat. 144:692-707.

Corresponding Editor: C. Boggs 